\title{
Índice de higiene oral simplificado en escolares Cuenca - Ecuador
}

\section{Simplified oral hygiene index in Cuenca schoolchildren - Ecuador}

\author{
Vintimilla, Katherine* y Encalada, Liliana \\ Universidad Católica de Cuenca. \\ Cuenca 010574, Ecuador. \\ *ktevin@hotmail.com
}

\begin{abstract}
Resumen
El objetivo principal de este estudio es determinar el Índice de Higiene Oral smplificado en escolares de 12 años, en la parroquia El Sagrario en la ciudad de Cuenca, Ecuador durante el año 2016. Es un estudio descriptivo retrospectivo, en el cual se evaluó 141 fichas epidemiológicas correspondientes a los escolares de 12 años de la parroquia "El Sagrario", las mismas que están archivadas en el Departamento de Investigación de la carrera de Odontología de la UCACUE. Se utilizaron los códigos de índice de higiene oral de Greene y Vermillion. La distribución de la muestra según el sexo fue $52 \%$ para el masculino y $48 \%$ en el femenino, se obtuvo un índice de 0,86 de placa blanda y un índice de 0,1 de placa calcificada dando mayor prevalencia en el sexo femenino, a su vez se demostró que el índice de higiene oral en la parroquia El Sagrario el de mayor prevalencia fue $61 \%$ de bueno higiene oral y el más bajo con $12 \%$ de excelente. El índice de higiene oral simplificado, estudiado en esta muestra indica que los escolares de 12 años de la parroquia El Sagrario, están dentro de los parámetros de una buena higiene oral, según la escala sugerida por Greene y Vermillion.
\end{abstract}

Palabras clave: Índice de higiene oral simplificada, índice de placa blanda, índice de placa calcificada, Greene Vermillion.

\begin{abstract}
The main objective of this study is to determine the Oral Hygiene Index in 12-year-old schoolchildren, in the El Sagrario parish in the city of Cuenca, Ecuador during 2016. It is a retrospective descriptive study, in which 141 index cards were evaluated Epidemiological data corresponding to the 12-year-old schoolchildren of the parish "El Sagrario", the same ones that are filed in the Research Department of the Dentistry of the UCACUE. The Greene and Vermillion oral hygiene index codes were used. The distribution of the sample according to sex was $52 \%$ for males and $48 \%$ for females, an index of 0.86 for soft plaque and an index of 0.1 for calcified plaque was obtained, giving a higher prevalence in sex In turn, the oral hygiene index in the El Sagrario parish was found to be $61 \%$ of good oral hygiene and the lowest with excellent 12 $\%$. The simplified oral hygiene index, studied in this sample, indicates that the 12-year-old schoolchildren of El Sagrario parish are within the parameters of good oral hygiene, according to the scale suggested by Greene and Vermillion.
\end{abstract}

Key words: Oral hygiene index, soft plaque, calcified plaque, Greene Vermillion.

\section{Introducción}

La higiene oral es uno de los aspectos más importantes de la salud integral de un individuo, y a su vez da a conocer las situaciones que llevan la enfermedad o salud de la cavidad bucal. ${ }^{12}$ La eliminación de la placa bacteriana se realizará por medio de métodos mecánicos y químicos, eliminando los microorganismos causantes de diferentes patologías orales. ${ }^{34}$

La placa dental es uno de los factores principales para el desarrollo de caries y enfermedad periodontal, si el tratamiento de estas no es inmediato o adecuado, puede derivar en complicaciones mayores dentro de la salud oral de un individuo, siendo la placa bacteriana uno de los signos que presenten en el $60 \%$ y $90 \%$ de la población escolar y adulta. ${ }^{45}$ Esto ha llamado mucho la atención en los últimos años, ya que los escolares son los que menos practican una buena higiene bucal, desarrollando así varias patologías bucodentales. ${ }^{6}$ Para prevenir estas patologías es indispensable mantener una higiene oral correcta a través del uso del cepillo dental (tres veces al día), hilo dental y enjuagues dentales. Sin embargo, cuando la placa no se elimina, desencadenará las patologías antes mencionadas. ${ }^{7}$

La placa bacteriana es una masa blanda y adherente de colonias bacterianas que se deposita sobre la superficie de los dientes, produciendo lesiones y enfermedad periodontal, siendo ambas las principales causantes de la pérdida dentaria. $^{89}$

Por esta razón, el propósito del presente estudio fue determinar el índice de higiene oral simplificado en escolares de 12 años de la parroquia El Sagrario. Para ello fue necesario obtener el respectivo consentimiento informado de los padres o tutores. Al finalizar el examen bucodental 
se le indicó el diagnóstico al paciente, motivándolo a que recupere su salud buco dental, tomando medidas preventivas más específicas para de esta manera mejorar su higiene oral.

\section{Materiales y Métodos}

El presente estudio fue exploratorio de corte transversal. Se procedió a revisar 141 fichas de escolares de la parroquia El Sagrario que contenían la información necesaria para este estudio, luego se ingresó las fichas en un sistema computarizado Epi Info 7.2, finalmente se procedió a tabular los datos y a realizar los respectivos análisis con los diferentes parámetros que se deseaban estudiar en esta investigación.

Para garantizar la confiabilidad en la recolección de los datos se realizó la calibración de los examinadores a fin de homogenizar conocimientos. A continuación por parte de la Universidad Católica de Cuenca se pidió permiso a través de una solicitud al Distrito Educativo Zonal 6, para que faciliten los datos de los estudiantes matriculados en cada uno de los Centros Educativos, se pidió autorización a los padres mediante el uso de un consentimiento informado y se les solicitó la aprobación de un asentimiento a los escolares para iniciar el examen clínico cubriendo de esta manera con los aspectos éticos y legales requeridos.

El estudio de índice de higiene oral busca describir cuantitativamente el problema en personas de 12 años de edad, utilizando el índice de Higiene Oral (OHI) de Greene y Vermillion para dientes permanentes. De esta manera las fichas originales del Mapa Epidemiológico de Salud Bucal de escolares de la ciudad de Cuenca 2016, que reposan en los archivos del departamento UCACUE, fueron llenadas con los siguientes criterios:

Empezaron por observar el 1er molar superior derecho (diente 1.6), continuando con el incisivo central superior (1.1), luego el 1er molar superior izquierdo $(2,6)$ y así sucesivamente se realizó en el cuadrante inferior, observando el 1er molar inferior izquierdo $(3,6)$, continuando con el incisivo central inferior $(3,1)$, y por último se analizó el 1er molar inferior derecho $(4,6)$.

Para el análisis de la Placa blanda se tomó en cuenta los siguientes códigos:

- Código 0 ausencia de placa blanda

- Código 1 Presencia de placa blanda cubriendo no más de $1 / 3$ de la superficie del diente.

- Código 2 Presencia de placa blanda cubriendo más de $1 / 3$, pero no más de $2 / 3$ de la superficie dental.

- Código 3 Presencia de placa blanda cubriendo más de 2/3 de la superficie dental.

De igual manera para el análisis de la placa calcificada se conoce los siguientes códigos:

- Código 0 Ausencia de cálculo.

- Código 1 Presencia de cálculo cubriendo no más de 1/3 de la superficie dental.

- Código 2 Presencia de cálculo cubriendo más de $1 / 3$, pero no más de 2/3 de la superficie dental.
- Código 3 Presencia de cálculo cubriendo más de 2/3 de la superficie dental.

Una vez analizado las piezas de cada estudiante para obtener el índice de higiene oral simplificado se suman los valores encontrados y se dividen entre las superficies examinadas, se realizó esta operación para ambos componentes. Luego de obtener el resultado de la suma, se procedió a determinar el grado clínico de higiene oral.

0.0 - 1.2: Buena higiene oral.

1.3 - 3.0: Higiene oral regular.

3.1 - 6.0: Mala higiene oral.

El levantamiento de datos se realizó entre los meses de septiembre y octubre del año 2016, mientras que el análisis de datos se realizó entre los meses de abril y mayo del 2017.

\section{Resultados}

El presente estudio se realizó con los datos epidemiológicos referentes al índice de higiene oral de los escolares de 12 años de la parroquia "El Sagrario", que consta en el archivo del Departamento de Investigación de la carrera de Odontología de la Universidad Católica de Cuenca, manifestando de los resultados siguientes:

Tabla 1. Distribución de la muestra de acuerdo al colegio y sexo en la Parroquia "El Sagrario"

\begin{tabular}{|c|c|c|c|c|c|c|}
\hline \multicolumn{7}{|l|}{ Unidades } \\
\hline \multirow[t]{2}{*}{ Educativas } & \multicolumn{2}{|c|}{ Femenino } & \multicolumn{2}{|c|}{ Masculino } & \multicolumn{2}{|c|}{ Total General } \\
\hline & $\mathrm{N}$ & $\%$ & $\mathrm{~N}$ & $\%$ & $\mathrm{~N}$ & $\%$ \\
\hline Ciudad De Cuenca & 13 & 9 & 4 & 3 & 17 & 12 \\
\hline Dolores. J. Torres & 42 & 30 & 6 & 4 & 48 & 34 \\
\hline Ezequiel Crespo & 6 & 4 & 26 & 18 & 32 & 23 \\
\hline Febres Cordero & 0 & 0 & 29 & 21 & 29 & 21 \\
\hline Otto Arosemena & 6 & 4 & 9 & 6 & 15 & 11 \\
\hline Total General & 67 & 48 & 74 & 52 & 141 & 100 \\
\hline
\end{tabular}

El estudio realizado en la parroquia "El Sagrario", contó con la colaboración de cinco instituciones educativas, con un total 141 escolares.

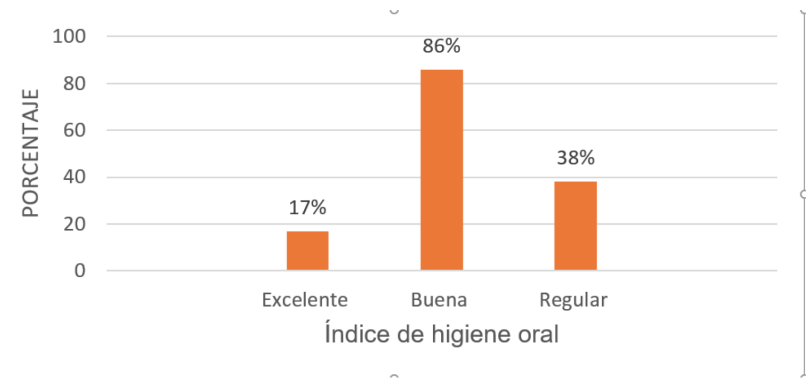

Fig. 1. Determinación del índice de higiene oral Simplificado en la Parroquia "El Sagrario"

En este gráfico (1) se puede observar el índice de higiene oral simplificado en la parroquia El Sagrario, representado en porcentaje. Sobresale el indicador de higiene oral buena 
con 86 , seguido por el regular con 38 y excelente cierra con 17.

Tabla 2. Índice de Higiene Oral Simplificado según el sexo

\begin{tabular}{|c|c|c|c|c|c|c|c|c|}
\hline Sexo & Excelente & & Buena & & Regular & & $\begin{array}{c}\text { Total } \\
\text { General }\end{array}$ & \\
\hline & $\mathbf{N}$ & 8 & $\mathbf{N}$ & $\%$ & $\mathbf{N}$ & $\%$ & $\mathbf{N}$ & $\%$ \\
\hline Femenino & 5 & 4 & 40 & 28 & 22 & 16 & 67 & 48 \\
\hline $\begin{array}{c}\text { Masculino } \\
\text { Total }\end{array}$ & 12 & 9 & 46 & 33 & 16 & 11 & 74 & 52 \\
\hline General & 17 & 12 & 86 & 61 & 38 & 27 & 141 & 100 \\
\hline
\end{tabular}

Se demostró la cantidad de escolares que acudieron a la revisión, donde 67 son del sexo femenino y 74 masculino. Se determinó que 5 tienen higiene oral excelente, 40 índice de higiene oral buena y 22 índice de higiene oral regular en mujeres mientras que de los varones 12 presentaron higiene oral excelente, 46 higiene oral buena y 16 higiene oral regular.

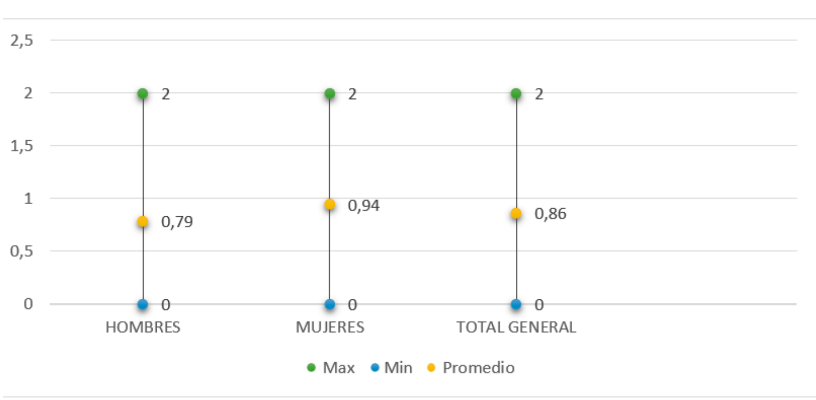

Fig. 2. Índice de la placa blanda comunitario según el sexo

El índice de placa blanda en escolares de 12 años en la parroquia "El Sagrario" fue ligeramente mayor en el sexo femenino que en el sexo masculino.

Los datos establecidos de I.P.B están dentro de los parámetros de una buena higiene oral.

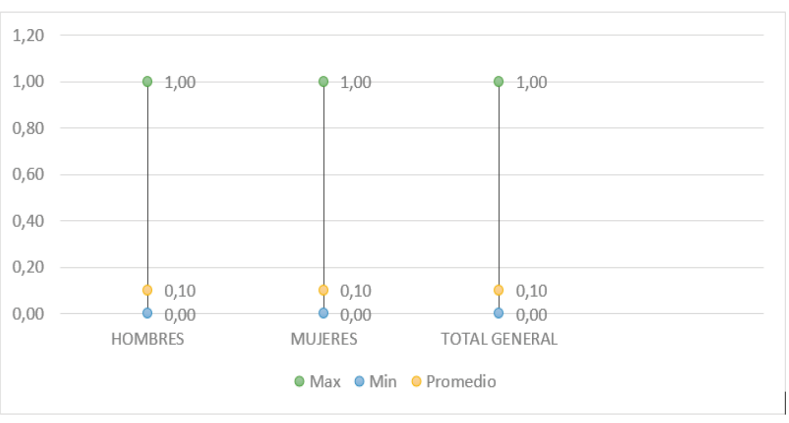

Fig. 3. Índice de placa calcificada comunitario según el sexo
El índice de placa calcificada en escolares de 12 años en la parroquia "El Sagrario" son similares tanto en el sexo masculino que en el sexo femenino.

Los datos establecidos de I.P.C. están dentro de los parámetros de una buena higiene oral.

\section{Discusión}

Para este estudio se tomaron las fichas de los escolares de 12 años de la parroquia "El Sagrario". Esta parroquia se encuentra localizada en el centro de la ciudad de Cuenca, los niños que acceden a los centros educativos de esta zona son de una economía estable, donde la principal fuente de ingresos de los padres es el comercio.

La muestra de la investigación fue de 141 fichas epidemiológicas con sus respectivos datos, distribuidos de acuerdo a sexo con 67 en mujeres y 74 varones fichas, esta muestra es similar al estudio de Ana Lagos Lagos en Lanquihue, Chile, ${ }^{10}$ quien analizaró escolares en ambos sexos.

El nivel de higiene oral tuvo su mayor porcentaje en el indicador "bueno" con $61 \%$, existiendo una mínima diferencia de acuerdo a sexo con $5 \%$ más para hombre que mujeres, resultados que son similares al estudio de Pazmiño Mendieta ${ }^{9}$ quien reportó luego de una revisión a 192 escolares de Machángará Cuenca que el nivel de higiene oral estuvo en un $76 \%$ en el indicador "bueno" dentro de la escala de Greene Vermillon. Esta semejanza puede deberse a que la población de estudio se encuentran en el mismo Cantón y presenta características demográficas, económicas y de educación similar a la muestra del presente estudio. A diferencia del estudio de Mediavilla Criollo $^{11}$ en el año 2011 en la fundación REMAR - Quito se evaluó a niños de 6 a 12 años dando un resultado con mayor porcentaje en el nivel de higiene oral "regular", tanto para los escolares de 6 años como los de 12 años, esta discrepancia de nivel con el presente estudio puede deberse a que los niños que se encuentran en REMAR son niños abandonados, huérfanos, consumidores de algunas sustancias lesivas para la salud general y bucal; sumado a una falta de interés en el cuidado dental y sobre todo una inadecuada técnica de limpieza bucodental, lo que puede estar en relación con el indicador de Higiene oral Regular.

Este estudio también reporto un índice comunitario de placa blanda de 0,86 y un índice de placa calcificada de 0.1 , valores que acreditan una buena higiene oral, para esta población de estudio, y esto se corrobora con el estudio de Pazmiño Mendieta 9 que reporto 0,7 en placa blanda y 0 en placa calcificada. A diferencia del estudio de Luis Mera Ramos? realizado en Quito en el año 2011, se presentó el índice de placa blanda 1,8 y el índice de placa calcificada con 0,01 , que de acuerdo al primer valor estaríamos hablando de una higiene "regular", aunque el indicador de placa calcificada sea óptimo. Hay que tomar en cuenta que la edad de 12 años no acredita a que presente placa calcificada debido a que la severidad de enfermedades periodontales 
es menos extensa en niños que en adultos con similares 11. Vega Ojeda DA. Índice de Higiene Oral en niños de 6 cantidades de placa bacteriana..

Los resultados obtenidos en el estudio demostraron que años de la parroquia Sayausí del cantón Cuenca, provincia Azuay-Ecuador 2016; ;

los escolares presentaban un índice de placa blanda ópti-12. Baruch Hernández NB, García Rivera ME. Eficacia de ma, con un mínimo de placa calcificada dando un nivel de higiene oral buena. Por lo tanto, es menester de las Universidades y más aún del MSP implementar sistemas 13. de prevención de patologías bucodentales para mejorar los indicadores de Higiene oral. la clorhexidina en el tratamiento de gingivitis:tabletas o colutorio. 2012-07;

3. Chica Gutiérrez RE. Eficacia del Propóleo al $25 \%$ vs. La Clorhexidina al $0.12 \%$ usado conjuntamente con técnica de Bass para disminuir la placa bacteriana.;

\section{Conclusiones}

14. Díaz Monteros C. Índice de higiene oral en escolares de 12 años, en la parroquia de San Joaquín del cantón Cuenca, provincia del Azuay - Ecuador. 2016.; 2016.

Con los resultados del estudio del índice de higiene oral 15 . Fuentes J, Bornhardt T. Prevalencia de Caries y Nivel de simplificado en escolares de 12 años en la Parroquia El Sagrario podemos concluir lo siguiente: Higiene Oral en Niños de 6 años Atendidos Bajo la Norma GES y el Modelo JUNAEB. 2014-12;8.

- El índice de higiene oral simplificado en la parroquia 16. Gil Loscos F. Periodoncia para el higienista dental. El Sagrario se encontró en el Nivel Bueno 2005;15.

- El índice de placa blanda comunitaria en los escolares 17. Gómez Ríos NI, Morales García MH. Determinación de de 12 años de la parroquia "El Sagrario" fue de 0,86 dando valores normales. los Índices CPO-D e IHOS en estudiantes de la Universidad Veracruzana, México. 2012;16.

- El índice de placa calcificada comunitaria fue de 0,1 18. Lagos Lagos A, Juárez Membreño IA. Necesidad de valores que se encuentra en los parámetros normales.

\section{Referencias Bibliográficas}

1. González Sanz nM, González Nieto BA, González Nieto E. Salud dental: relación entre la caries dental y el consumo de alimentos. 2013-07;28.

2. Pazmiño Mendieta DK. Índice de Higiene Oral en niños de 12 años de edad en las Escuelas de la parroquia "Machángara" de la Ciudad de Cuenca, en el Periodo Escolar 2016.;

3. L M. Índice de higiene oral simplificado comparativo entre niños y niñas de 6 a 12 años de edad, de la escuela fiscal "Himmelman" y la unidad educativa particular "Héroes del Cenepa" del cantón Cayambe de la provincia de Pichincha, en el periodo 2010-2011.;

4. Fernández González C, Núñez Franz L, Díaz Sanzana N. Determinantes de salud oral en población de 12 años. 2011;4.

5. Gómez Osorno AM, Bernal Álvarez T, Posada López A. tratamiento periodontal e higiene oral en adolescentes de 12 años de Llanquihue. 2014;7.

19. Mediavilla Criollo FI. Determinacion del indice de higiene oral simplificado en niños y niñas de 6 a 12 años de edad de la fundacion remar - quito, en el mes de julio del año 2011.;

20. Naverac Aznar M, de Grado Cabanilles P. Periodoncia para el Higienita dental. 2007;17.

1. Pulido Rozo M, Gonzalez Martínez F. Enfermedad periodontal e indicadores de higiene bucal en estudiantes de secundaria Cartagena, Colombia. 2011-09;13.

22. Serrano-Granger J. La placa dental como biofilm.¿Cómo eliminarla? 2005-08;

Recibido: 3 de julio de 2017

Aceptado: 7 de agosto de 2017 en población de 3 a 5 años de una institución educativa de Medellín y sus factores relacionados. 2015;11.

6. Barranca Enríquez A. Manual de Técnicas de Higiene Oral; 2011.

7. Díaz Caballero AJ, Fonseca Ricaurte MA. Cálculo dental una revisión de literatura y presentación de una condición inusual. 2011;49.

8. Corchuelo J. Sensibilidad y especificidad de un índice de higiene oral de uso comunitario. 2011-10;42.

9. Nivelo EA. Frecuencia de la Buena Higiene Oral en niños de 12 años de edad en las Escuelas de la Parroquia "El Batán" de la ciudad de Cuenca 2016.;

10. Villavicencio León AE. Índice de Higiene Oral Simplificado en niños de 12 años en la parroquia Cañaribamba del cantón Cuenca, provincia del Azuay, Ecuador, 2016;. 